\title{
General Integrable Problems of Classical Mechanics
}

\section{O.I. Bogoyavlenskij ${ }^{\star}$}

The Fields Institute for Research in Mathematical Sciences, Waterloo, Ontario, Canada, N2L 5Z5 and Queen's University, Kingston, Ontario, Canada, K7L 3N6

Received April 4, 1992; in revised form June 29, 1992

\begin{abstract}
Several classical problems of mechanics are shown to be integrable for the special systems of coupled rigid bodies, introduced in this work and called $C^{k}$-central configurations. It is proven that dynamics of an arbitrary $C^{k}$-central configuration in a Newtonian gravitational field with an arbitrary quadratic potential is integrable in the Liouville sense and in the theta-functions of Riemann surfaces. Hidden symmetry of the inertial dynamics of these configurations is disclosed and reductions of the Lagrange equations to the Euler equations on Lie coalgebras are obtained. Reductions and integrable cases of a heavy $C^{k}$-central configuration rotation around a fixed point are indicated. Separation of rotations of a space station type orbiting system, being a $C^{k}$-central configuration of rigid bodies, is proven. This result leads to the possibility of the independent stabilization of rotations of the rigid bodies in such orbiting configurations.
\end{abstract}

\section{Contents}

1. Introduction and Summary . . . . . . . . . . . . . . . . . 24

2. Complete integrability of dynamics of a $C^{1}$-central configuration. . . . . 25

3. General integrable problems of classical mechanics . . . . . . . . . . . . 29

4. Hidden symmetry of the inertial dynamics. . . . . . . . . . . . . 31

5. Reductions and integrable cases of $C^{k}$-central configuration rotation around a fixed point in the Newtonian gravitational fields with quadratic

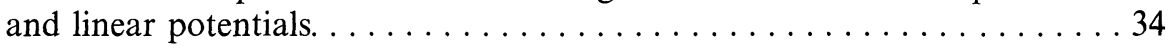

6. Multibody integrable generalization of the Neumann problem . . . . . 35

7. Separation of rotations of an orbiting space station type $C^{k}$-central

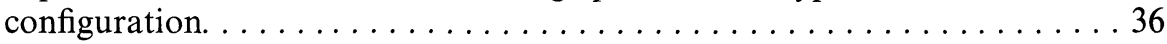

8. Separation of rotations of $C R^{n}$-central configuration of coupled gyrostats .40

\footnotetext{
* Supported by the Ministry of Colleges and Universities of Ontario and the Natural Sciences and Engineering Research Council of Canada
} 


\section{Introduction and Summary}

Recently Sreenath, Oh, Krishnaprasad and Marsden [25] investigated equilibria and stability of coupled rigid bodies by the energy-Casimir method and applied the Poincaré-Melnikov method to prove the stochastization of dynamics under small perturbations of homoclinic orbits. In the work [25] also the integrability of the inertial dynamics of two coupled planar (two-dimensional) rigid bodies is proven after indicating a reduction to a Hamiltonian system with one degree of freedom. Reductions, symmetry and phases in general problems of coupled rigid bodies dynamics were studied by Marsden, Montgomery and Ratiu [16]. The energymomentum method and block diagonalization method were developed by Marsden, Simo, Lewis and Posbergh [15] and by Lewis, Marsden, Ratiu and Simo [14]. Applications of these methods to the coupled rigid bodies dynamics were considered by Krishnaprasad and Marsden [11], Krishnaprasad [12] and Patrick $[21,22]$. Equilibrium rotations of planar kinematic chains of (two-dimensional) rigid bodies were studied by Baillieul [3]. Integrable cases of rotations of two multi-dimensional interacting (but not coupled) rigid bodies were constructed by Bobenko, Reyman and Semenov-Tian-Shansky [4]. Dynamics of orbiting multibody configurations, including rigid and flexible bodies was studied by Modi and Suleman [17] and by Modi, Ng, Suleman and Morita [18]. General problems of coupled rigid bodies dynamics were considered in monographs by Routh [23], Leimanis [13] and Wittenburg [26].

The present work is devoted to the construction and investigation of integrable cases of three-dimensional coupled rigid bodies dynamics. We introduce the $C^{k}$-central configurations of rigid bodies, coupled by the ideal spherical ballin-socket or hinge joints or by the Cardan suspensions without torque and friction. These configurations have tree structures with the main rigid body $T_{0 k}$ chosen, which is coupled with many branchy chains of rigid bodies, having lengths not greater than $k$, and without closed chains.

The rigid bodies in the $C^{k}$-central configurations are arbitrary, with arbitrary distributions of masses and inertia tensors. The spherical joints are placed in such a way, that the following mechanical property is fulfilled: If we fix the main rigid body $T_{0 k}$ and rotate all other rigid bodies independently around their spherical joints, then the mass center of the whole configuration does not move. The mechanical property is equivalent to the constructive definition of $C^{k}$-central configuration, presented in Sects. 2 and 3.

If one chooses an arbitrary system $S$ of coupled rigid bodies, having tree structure without closed chains, then after appropriate shifts of spherical joints one obtains the $C^{k}$-central configuration. Therefore the dynamics of an arbitrary system $S$ of coupled rigid bodies can be studied as perturbation of dynamics of an appropriate $C^{k}$-central configuration.

Our main theorem, proven in Sects. 2 and 3 states that dynamics of an arbitrary $C^{k}$-central configuration in the Newtonian gravitational field with an arbitrary quadratic potential is integrable in the Liouville sense and in the theta-functions of Riemann surfaces. This theorem holds also for dynamics of coupled $n$-dimensional rigid bodies $(n=2,3,4, \ldots)$ in $n$-dimensional Euclidean space and has the same proof of all $n \geqq 2$. Here we have infinitely many arbitrary parameters, connected with the inertia tensors of the rigid bodies and concrete choice of graph of the tree structure and arbitrary form of the Newtonian quadratic potential. Therefore our main theorem describes the general integrable problems of classical mechanics. 
The integrability of the problem considered is based on the fundamental physical law of the identity of the inertial and gravitational masses. Gravitational force, acting on a material point in the Newtonian gravitational field, is proportional to its mass. Therefore the kinetic energy and the potential energy of the $C^{k}$-central configuration depend on the same parameters, which are the entries of the inertia tensors of the coupled rigid bodies. Equations of rotation of even a single electrically charged rigid body in the electrostatic field with an arbitrary quadratic potential are not integrable.

In Sect. 4 a hidden symmetry of the inertial dynamics of an arbitrary $C^{k}$-central configuration is disclosed and reductions of the Lagrange equations to the Euler equations on the direct sums of Lie coalgebras are pointed out. In Sect. 5 reductions and integrable cases of rotation of a $C^{k}$-central configuration around a fixed point in Newtonian gravitational fields with quadratic and linear potentials are found. Multibody integrable generalization of the classical Neumann problem is presented in Sect. 6.

The problem of rotation around the mass center of a space station type orbiting system, being a $C^{k}$-central configuration of rigid bodies, is studied in Sect. 7. Separation of rotations of the rigid bodies in such a configuration is proven. The corresponding Lagrange equations are embedded into the Euler equations on the direct sums of the Lie coalgebras, which are autonomous in the case of the circular orbit and nonautonomous for the general elliptic orbit of the mass center.

Dynamics of more general $C R^{n}$-central configurations of coupled gyrostats, having all possible joints of rigid bodies: spherical or ball-in-socket joints, pin joints and universal joints, is studied in Sect. 8. Separation of rotations of an orbiting space station type $C R^{n}$-central configuration is obtained and integrability of inertial dynamics is proven.

\section{Complete Integrability of Dynamics of a $C^{1}$-Central Configuration}

I. Let us consider dynamics of a system $S$ of $n+1$ rigid bodies, consisting of the main rigid body $T_{0}$ and $n$ rigid bodies $T_{\alpha}(\alpha=1, \ldots, n)$. Rigid body $T_{0}$ is coupled with each rigid body $T_{\alpha}$ in its $\left(T_{\alpha}\right)$ mass center $\mathbf{P}_{\alpha}$ by the ideal spherical ballin-socket or hinge joint or by the Cardan suspension, so they are free to rotate relative to each other. Particles of rigid body $T_{0}$ have Lagrange coordinates $r_{0}^{i}$. Particles of rigid body $T_{\alpha}$ have Lagrange coordinates $r_{\alpha}^{i}$; its mass center $\mathbf{P}_{\alpha}$ is in the origin $(0,0,0)$ of coordinate system $r_{\alpha}^{i}$. The center of the spherical joint $\mathbf{P}_{\alpha}$ in the rigid body $T_{0}$ has Lagrange coordinates $r_{0 \alpha}^{i}$, which are arbitrary. Euler coordinates of the points $\mathbf{P}_{\alpha}$ are $q_{\alpha}^{1}(t), q_{\alpha}^{2}(t), q_{\alpha}^{3}(t)$.

Let $T$ denote an effective rigid body, which is obtained from the rigid body $T_{0}$ by putting into the points $\mathbf{P}_{\alpha}$ masses $m_{\alpha}$, equal to the masses of the rigid bodies $T_{\alpha}$. Lagrange coordinates $r_{0}^{i}$ are also used for the effective rigid body $T$; we choose them in such a way that the mass center $\mathbf{P}$ of the effective rigid body $T$ has Lagrange coordinates $r_{0}^{i}=0$. Euler coordinates of the mass center $\mathbf{P}$ are $q^{i}(t)$. Let $\rho_{0}\left(r_{0}^{1}, r_{0}^{2}, r_{0}^{3}\right)$ and $\rho_{\alpha}\left(r_{\alpha}^{1}, r_{\alpha}^{2}, r_{\alpha}^{3}\right)$ be the mass densities of the rigid bodies $T_{0}$ and $T_{\alpha}$.

We shall call such system $S$, composed of the main rigid body $T_{0}$, coupled with $n$ rigid bodies $T_{1}, \ldots, T_{n}$ in their mass centers $\mathbf{P}_{\alpha}$ as the $C^{1}$-central configuration. The main mechanical property of the $C^{1}$-central configuration is the following: if we fix the main rigid body $T_{0}$ and rotate independently and arbitrarily all rigid bodies $T_{1}, \ldots, T_{n}$ around their spherical joints (which coincide with their mass 
centers $\mathbf{P}_{\alpha}$ ) then the mass center $\mathbf{P}$ of the whole configuration does not move. The proof obviously consists in verifying that the mass center of the system $S$ coincides with the mass center of the effective rigid body $T$, which is fixed.

The configuration space $M$ of the system $S$ is the direct product of Lie groups

$$
M=R^{3} \times \prod_{\alpha=0}^{n} S O(3)_{\alpha} .
$$

Position of the mass center $\mathbf{P}$ is determined by the vector $\mathbf{q}(t) \in R^{3}$, rotation of the effective rigid body $T$ around its mass center $\mathbf{P}$ is determined by the orthogonal matrix $Q_{j}^{i}(t) \in S O(3)_{0}$, rotation of the rigid body $T_{\alpha}$ around its mass center $\mathbf{P}_{\alpha}$ is determined by the orthogonal matrix $Q_{\alpha j}^{i}(t) \in S O(3)_{\alpha}$.

Euler coordinates of particles of the rigid body $T_{0}$ are determined by the formulae

$$
x^{i}=\sum_{j=1}^{3} Q_{j}^{i}(t) r_{0}^{j}+q^{i}(t) .
$$

Euler coordinates of particles of the rigid body $T_{\alpha}$ are determined by the expressions

$$
x_{\alpha}^{i}=\sum_{j=1}^{3} Q_{\alpha j}^{i}(t) r_{\alpha}^{j}+q_{\alpha}^{i}(t),
$$

where $q_{\alpha}^{1}(t), q_{\alpha}^{2}(t), q_{\alpha}^{3}(t)$ are Euler coordinates of the center of the spherical joint $\mathbf{P}_{\alpha}$, which belongs also to the rigid body $T_{0}$. In view of (2.2) we have

$$
q_{\alpha}^{i}(t)=\sum_{j=1}^{3} Q_{j}^{i}(t) r_{0 \alpha}^{j}+q^{i}(t) .
$$

II.

Theorem 1. Dynamics of an arbitrary $C^{1}$-central configuration $S^{1}$ in the Newtonian gravitational field with an arbitrary quadratic potential

$$
\varphi\left(x^{1}, x^{2}, x^{3}\right)=\frac{1}{2} \sum_{i, j=1}^{3}\left(a_{i j} x^{i} x^{j}+b_{i} x^{i}\right)
$$

is described by a Hamiltonian system which is completely integrable in the Liouville sense. Dynamics of the mass center $\mathbf{P}$ is integrable in terms of elementary functions. Rotations of the rigid bodies $T, T_{1}, \ldots, T_{n}$ around their mass centers $\mathbf{P}, \mathbf{P}_{1}, \ldots, \mathbf{P}_{n}$ take place independently and are integrable in terms of the theta-functions of Riemann surfaces.

Proof. The Lagrangian of the $C^{1}$-central configuration $S^{1}$ in the fixed system of reference $F$ is equal to the integral with respect to the volumes of all rigid bodies $T_{0}, T_{1}, \ldots, T_{n}$ of Lagrange functions of their particles:

$$
\begin{gathered}
L_{S}=L_{0}+\sum_{\alpha=1}^{n} L_{\alpha} \\
L_{0}=\frac{1}{2} \int_{T_{0}} \rho_{0}\left(\mathbf{r}_{0}\right)\left(\dot{x}_{0}, \dot{x}_{0}\right) d^{3} \mathbf{r}_{0}-\int_{T_{0}} \rho_{0}\left(\mathbf{r}_{0}\right) \varphi\left(x_{0}\right) d^{3} \mathbf{r}_{0}
\end{gathered}
$$




$$
\begin{gathered}
L_{\alpha}=\frac{1}{2} \int_{T_{\alpha}} \rho_{\alpha}\left(\mathbf{r}_{\alpha}\right)\left(\sum_{i=1}^{3}\left(\sum_{j=1}^{3} \dot{Q}_{\alpha j}^{i} r_{\alpha}^{j}+\dot{q}_{\alpha}^{i}\right)^{2}\right) d^{3} \mathbf{r}_{\alpha} \\
-\int_{T_{\alpha}} \rho_{\alpha}\left(\mathbf{r}_{\alpha}\right) \varphi\left(\sum_{j=1}^{3} Q_{\alpha j}^{1} r_{\alpha}^{j}+q_{\alpha}^{1}, \sum_{j=1}^{3} Q_{\alpha j}^{2} r_{\alpha}^{j}+q_{\alpha}^{2}, \sum_{j=1}^{3} Q_{\alpha j}^{3} r_{\alpha}^{j}+q_{\alpha}^{3}\right) d^{3} \mathbf{r}_{\alpha} .
\end{gathered}
$$

Lagrange coordinates $r_{\alpha}^{i}$ are chosen in such a way that mass center $\mathbf{P}_{\alpha}$ has zero coordinates, so the equalities hold

$$
\int_{T_{\alpha}} \rho_{\alpha}\left(\mathbf{r}_{\alpha}\right) r_{\alpha}^{j} d^{3} \mathbf{r}_{\alpha}=0
$$

In view of the definition of the inertia tensor $I_{\alpha i k}$ we have

$$
\begin{aligned}
\int_{T_{\alpha}} \rho_{\alpha}\left(\mathbf{r}_{\alpha}\right) r_{\alpha}^{i} r_{\alpha}^{j} d^{3} \mathbf{r}_{\alpha} & =\frac{1}{2} \operatorname{Tr}\left(I_{\alpha}\right) \delta_{i j}-I_{\alpha i j} \\
& =J_{\alpha i j} .
\end{aligned}
$$

Expression (2.7) after substituting formulae (2.4) and integrating with respect to the volume of the rigid body $T_{\alpha}$ takes the form

$$
\begin{aligned}
L_{\alpha} & =L_{1 \alpha}+L_{2 \alpha}, \\
L_{1 \alpha} & =\frac{1}{2} m_{\alpha}\left(\dot{\mathbf{q}}_{\alpha}, \dot{\mathbf{q}}_{\alpha}\right)-m_{\alpha} \varphi\left(\mathbf{q}_{\alpha}\right), \\
L_{2 \alpha} & =\frac{1}{2} \sum_{i, j, k=1}^{3} \dot{Q}_{\alpha j}^{i} J_{\alpha j k} \dot{Q}_{\alpha k}^{i}-\frac{1}{2} \sum_{i, j, k, m=1}^{3} a_{i j} Q_{\alpha k}^{i} Q_{\alpha m}^{j} J_{\alpha k m} \\
& =-\frac{1}{2} \operatorname{Tr}\left(J_{\alpha} Q_{\alpha}^{-1} \dot{Q}_{\alpha} Q_{\alpha}^{-1} \dot{Q}_{\alpha}\right)+\frac{1}{2} \operatorname{Tr}\left(Q_{\alpha}^{t} a Q_{\alpha} I_{\alpha}\right),
\end{aligned}
$$

where additive constants are omitted. In this calculation we used essentially the quadratic forms of the kinetic energy and of the Newtonian potential (2.4). In Lagrangian (2.7) all linear with respect to $r_{\alpha}^{i}$ summands are equal to zero after the integration in view of the equalities (2.8).

From the formulae (2.5) and (2.10) we receive

$$
L_{S}=L_{T}+\sum_{\alpha=1}^{n} L_{2 \alpha},
$$

where Lagrangian $L_{T}$ in view of (2.6) and (2.11) has the form

$$
\begin{aligned}
L_{\boldsymbol{T}}= & L_{0}+\sum_{\alpha=1}^{n} L_{\alpha 1} \\
= & \frac{1}{2} \int_{T_{0}} \rho_{0}\left(\mathbf{r}_{0}\right)\left(\dot{\mathbf{x}}_{0}, \dot{\mathbf{x}}_{0}\right) d^{3} \mathbf{r}_{0}-\int_{T_{0}} \rho_{0}\left(\mathbf{r}_{0}\right) \varphi\left(\mathbf{x}_{0}\right) d^{3} \mathbf{r}_{0} \\
& +\frac{1}{2} \sum_{\alpha=1}^{n} m_{\alpha}\left(\dot{\mathbf{q}}_{\alpha}, \dot{\mathbf{q}}_{\alpha}\right)-\sum_{\alpha=1}^{n} m_{\alpha} \varphi\left(\mathbf{q}_{\alpha}\right) .
\end{aligned}
$$

Vector $\mathbf{q}_{\alpha}(t)$ describes the dynamics of the center of the spherical joint $\mathbf{P}_{\alpha}$, which by definition of the $C^{1}$-central configuration $S^{1}$ belongs to the rigid body $T_{0}$ (and 
$T_{\alpha}$ also). Therefore the function $L_{T}$ is the Lagrangian of the effective rigid body $T$, having masses $m_{\alpha}$ in the points $\mathbf{P}_{\alpha}$. The mass density $\rho\left(\mathbf{r}_{0}\right)$ of the effective rigid body $T$ is determined by the formula

$$
\rho\left(\mathbf{r}_{0}\right)=\rho_{0}\left(\mathbf{r}_{0}\right)+\sum_{\alpha=1}^{n} m_{\alpha} \delta\left(\mathbf{r}_{0}-\mathbf{P}_{\alpha}\right) .
$$

So Lagrangian (2.14) takes the form

$$
L_{T}=\frac{1}{2} \int_{T} \rho\left(\mathbf{r}_{0}\right)\left(\dot{\mathbf{x}}_{0}, \dot{\mathbf{x}}_{0}\right) d_{3} \mathbf{r}_{0}-\int_{T} \rho\left(\mathbf{r}_{0}\right) \varphi\left(\mathbf{x}_{0}\right) d_{3} \mathbf{r}_{0} .
$$

Substituting here the formulae (2.2), we get

$$
\begin{aligned}
L_{T}= & \frac{1}{2} \int_{T} \rho\left(\mathbf{r}_{0}\right) \sum_{i=1}^{3}\left(\sum_{j=1}^{3} \dot{Q}_{j}^{i} r_{0}^{j}+\dot{q}^{i}\right)^{2} d^{3} \mathbf{r}_{0} \\
& -\int_{T} \rho\left(\mathbf{r}_{0}\right) \varphi\left(\sum_{j=1}^{3} Q_{j}^{1} r_{0}^{j}+q^{1}, \sum_{j=1}^{3} Q_{j}^{2} r_{0}^{j}+q^{2}, \sum_{j=1}^{3} Q_{j}^{3} r_{0}^{j}+q^{3}\right) d^{3} \mathbf{r}_{0} .
\end{aligned}
$$

In view of the definitions of the inertia tensor $I_{i k}$ and mass center of the effective rigid body $T$ the formulae are valid

$$
\begin{aligned}
\int_{T} \rho\left(\mathbf{r}_{0}\right) r_{0}^{i} r_{0}^{j} d^{3} \mathbf{r}_{0} & =\frac{1}{2} \operatorname{Tr}(I) \delta_{i j}-I_{i j}=J_{i j}, \\
\int_{T} \rho\left(\mathbf{r}_{0}\right) r_{0}^{i} d^{3} \mathbf{r}_{0} & =0 .
\end{aligned}
$$

Expression (2.17) after substituting the formula (2.4) and integrating with respect to the volume of the rigid body $T$ in view of (2.18) takes the form

$$
\begin{aligned}
& L_{T}=L_{1}+L_{2}, \\
& L_{1}=\frac{1}{2} m(\dot{\mathbf{q}}, \dot{\mathbf{q}})-m \varphi(\mathbf{q}), \\
& L_{2}=-\frac{1}{2} \operatorname{Tr}\left(J Q^{-1} \dot{Q} Q^{-1} \dot{Q}\right)+\frac{1}{2} \operatorname{Tr}\left(Q^{t} a Q I\right) .
\end{aligned}
$$

Here $m$ is the mass of the effective rigid body $T$, or total mass of all rigid bodies $T_{0}, T_{1}, \ldots, T_{n}$.

Thus, in view of (2.13) we receive the resulting formula for the Lagrangian $L_{S^{1}}$ of the $C^{1}$-central configuration $S^{1}$ :

$$
L_{S^{1}}=L_{1}(\mathbf{q}, \dot{\mathbf{q}})+L_{2}(Q, \dot{Q})+\sum_{\alpha=1}^{n} L_{2 \alpha}\left(Q_{\alpha}, \dot{Q}_{\alpha}\right) .
$$

Time evolution of vector $\mathbf{q}$ and $n+1$ orthogonal matrices $Q, Q_{\alpha}(\alpha=1, \ldots, n)$ describes completely the dynamics on the configuration manifold $M(2.1)$, that is dynamics of the system of coupled rigid bodies $S^{1}$. The basic formula (2.22) shows that variables $\mathbf{q}, Q, Q_{\alpha}$ are separated and there is no interaction between dynamics of the mass center $\mathbf{P}$ and rotation of the rigid bodies $T, T_{\alpha}$ around their mass centers $\mathbf{P}$ and $\mathbf{P}_{\alpha}$. 
The Lagrangian $L_{1}(2.20)$ in view of (2.4) is the Lagrangian of a harmonic oscillator. So the dynamics of the mass center $\mathbf{P}$ of the system $S^{1}$ is integrable in terms of elementary functions.

The Lagrangians $L_{2}(2.21)$ and $L_{2 \alpha}(2.12)$ describe rotation of a rigid body around a fixed point in the gravitational field with the homogeneous quadratic potential (without linear terms). This problem is studied in detail by Bogoyavlenskij $[6,7]$, where it is proven that the corresponding Hamiltonian system is integrable in the Liouville sense and dynamics is integrable in terms of the theta-functions of the Riemann surfaces. (History of this problem, its generalizations and bibliography are presented in survey [10]). Therefore dynamics of the $C^{1}$-central configuration $S^{1}$ also is integrable in the Liouville sense and in the Riemann theta-functions. Theorem 1 is proven.

Remark 1. In Bogoyavlenskij [8, 9, 10] the integrability of dynamics of one arbitrary rigid body (without a fixed point) in a Newtonian gravitational field with an arbitrary quadratic potential is proven. This is the special case of Theorem 1.

\section{General Integrable Problems of Classical Mechanics}

The main consequence of the Theorem 1 is that the Lagrangian of an arbitrary $C^{1}$-central configuration $S^{1}$ in the Newtonian gravitational field with an arbitrary quadratic potential (2.4) is split into the sum (2.22), where the vector $\mathbf{q}(t)$ stays for the mass center of all system $S^{1}$.

We consider $m C^{1}$-central configurations denoted as $S_{1}^{1}, \ldots, S_{m}^{1}$ and let $\mathbf{q}_{1}^{1}(t), \ldots, \mathbf{q}_{m}^{1}(t)$ be vectors of their mass centers. We call the $C^{2}$-central configuration a system of rigid bodies, obtained by the spherical coupling of the main rigid body $T_{02}$ with $m C^{1}$-central configurations $S_{1}^{1}, \ldots, S_{m}^{1}$ in their mass centers $\mathbf{q}_{1}^{1}, \ldots, \mathbf{q}_{m}^{1}$. The effective rigid body $T_{2}$ is obtained from the rigid body $T_{02}$ by putting masses $m_{i}^{1}$ (the mass of the system $S_{i}^{1}$ ) into the points $\mathbf{q}_{i}^{1}$.

By induction we call $C^{k}$-central configuration a system of rigid bodies, obtained by coupling the main rigid body $T_{0 k}$ with $n C^{k-1}$-central configurations $S_{1}^{k-1}, \ldots, S_{n}^{k-1}$ in their mass centers $\mathbf{q}_{1}^{k-1}, \ldots, \mathbf{q}_{n}^{k-1}$. The effective rigid body $T_{k}$ is obtained from the rigid body $T_{0 k}$ by putting masses $m_{i}^{k-1}$ (mass of the system $S_{i}^{k-1}$ ) into the points $\mathbf{q}_{i}^{k-1}$. The mass center $\mathbf{P}_{k}$ of $C^{k}$-central configuration $S^{k}$ coincides with the mass center of the effective rigid body $T_{k}$. Therefore the mass center $\mathbf{P}_{k}$ does not move if all rigid bodies in this configuration (except $T_{0 k}$ ) arbitrarily rotate around their spherical joints.

Theorem 2. Dynamics of an arbitrary $C^{k}$-central configurations $S^{k}$ of $n$ coupled rigid bodies in the Newtonian gravitational field with an arbitrary quadratic potential (2.4) is described by the Hamiltonian system which is completely integrable in the Liouville sense. The Lagrangian $L_{S^{k}}$ of the $C^{k}$-central configuration $S^{k}$ is split into the sum

$$
\begin{aligned}
L_{S^{k}} & =L_{1}(\mathbf{q}, \dot{\mathbf{q}})+\sum_{\alpha=1}^{N} L_{2 \alpha}\left(Q_{\alpha}, \dot{Q}_{\alpha}\right), \\
L_{1}(\mathbf{q}, \dot{\mathbf{q}}) & =\frac{1}{2} m(\dot{\mathbf{q}}, \dot{\mathbf{q}})-m \varphi(\mathbf{q}), \\
L_{2 \alpha}\left(Q_{\alpha}, \dot{Q}_{\alpha}\right) & =-\frac{1}{2} \operatorname{Tr}\left(J_{\alpha} Q_{\alpha}^{-1} \dot{Q}_{\alpha} Q_{\alpha}^{-1} \dot{Q}_{\alpha}\right)+\frac{1}{2} \operatorname{Tr}\left(Q_{\alpha}^{t} a Q_{\alpha} I_{\alpha}\right),
\end{aligned}
$$


where vector $\mathbf{q}(t)$ stays for the mass center of the configuration $S^{k}$ (with the whole mass $m$ ) and the orthogonal matrix $Q_{\alpha}$ describes rotation of the $\alpha$-th effective rigid body around its mass center. Dynamics of mass center $\mathbf{q}(t)$ is integrable in terms of elementary functions. Rotation of each rigid body $T_{\alpha}$ is integrable in terms of the Riemann theta-functions.

Proof. Theorem 2 for the $C^{1}$-central configurations is proven in Sect. 2. Therefore let us suppose by induction the theorem is proven for $C^{k-1}$-central configurations $S_{1}^{k-1}, \ldots, S_{n}^{k-1}$. That means their Lagrangians $L_{S_{j}^{k-1}}$ have the form:

$$
\begin{aligned}
L_{S_{j}^{k-1}} & =L_{1 j}\left(\mathbf{q}_{j}, \dot{\mathbf{q}}_{j}\right)+\sum_{\alpha=1}^{n_{j}} L_{2 \alpha}\left(Q_{\alpha}, \dot{Q}_{\alpha}\right), \\
L_{1 j} & =\frac{1}{2} m_{j}\left(\dot{\mathbf{q}}_{j}, \dot{\mathbf{q}}_{j}\right)-m_{j} \varphi\left(\mathbf{q}_{j}\right)
\end{aligned}
$$

where vector $\mathbf{q}_{j}(t)$ stays for the mass center of the configuration $S_{j}^{k-1}$ (with the whole mass $m_{j}$ ). The $C^{k}$-central configuration $S^{k}$ is obtained by the spherical coupling of the main rigid body $T_{0 k}$ in the points $\mathbf{P}_{j}$ with the $C^{k-1}$-central configurations $S_{j}^{k-1}(j=1, \ldots, n)$ in their mass centers $\mathbf{q}_{j}$. The Lagrangian $L_{S^{k}}$ of the configuration $S^{k}$ has the form

$$
L_{S^{k}}=L_{T_{0 k}}+\sum_{j=1}^{n} L_{S_{j}^{k-1}}
$$

The Lagrangian $L_{T_{0 k}}$ is given by the formula

$$
\begin{aligned}
L_{T_{0 k}}= & \frac{1}{2} \int_{T_{0 k}} \rho_{0}\left(\mathbf{r}_{0}\right) \sum_{i=1}^{3}\left(\sum_{j=1}^{3} \dot{Q}_{j}^{i} r_{0}^{j}+\dot{q}^{i}\right)^{2} d^{3} \mathbf{r}_{0} \\
& -\int_{T_{0 k}} \rho_{0}\left(\mathbf{r}_{0}\right) \varphi\left(\sum_{j=1}^{3} Q_{j}^{1} r_{0}^{j}+q^{1}, \sum_{j=1}^{3} Q_{j}^{2} r_{0}^{j}+q^{2}, \sum_{j=1}^{3} Q_{j}^{3} r_{0}^{j}+q^{3}\right) d^{3} \mathbf{r}_{0} .
\end{aligned}
$$

Here $\left(r_{0}^{1}, r_{0}^{2}, r_{0}^{3}\right)$ are Lagrange coordinates of particles of the rigid body $T_{0 k}, \rho_{0}\left(\mathbf{r}_{0}\right)$ is its mass density. Vector $\left(q^{1}, q^{2}, q^{3}\right)$ stays for the mass center of the effective rigid body $T_{k}$ and coincides with the mass center of the whole configuration $S^{k}$. The effective rigid body $T_{k}$ is obtained from the rigid body $T_{0 k}$ by putting masses $m_{j}$ into the coupling points $\mathbf{P}_{j}\left(\mathbf{q}_{j}\right)$. Its mass density has the form

$$
\rho\left(\mathbf{r}_{0}\right)=\rho_{0}\left(\mathbf{r}_{0}\right)+\sum_{j=1}^{n} m_{j} \delta\left(\mathbf{r}_{0}-\mathbf{P}_{j}\right) .
$$

Orthogonal matrix $Q_{0}$ describes rotation of the effective rigid body $T_{k}$ around its mass center $\mathbf{q}$.

Formula (3.5) after substituting the expressions (3.4), (3.6) and using the formula (3.7) is transformed into

$$
L_{S^{k}}=L_{T_{k}}+\sum_{j=1}^{n} \sum_{\alpha=1}^{n_{j}} L_{2 \alpha}\left(Q_{\alpha}, \dot{Q}_{\alpha}\right) .
$$


Here the Lagrangian $L_{T_{k}}$ of the effective rigid body $T_{k}$ has the form

$$
\begin{aligned}
L_{T_{k}}= & \frac{1}{2} \int_{T_{k}} \rho\left(\mathbf{r}_{0}\right) \sum_{i=1}^{3}\left(\sum_{j=1}^{3} \dot{Q}_{j}^{i} r_{0}^{j}+\dot{q}^{i}\right)^{2} d^{3} \mathbf{r}_{0} \\
& -\int_{T_{k}} \rho\left(\mathbf{r}_{0}\right) \varphi\left(\sum_{j=1}^{3} Q_{j}^{1} r_{0}^{j}+q^{1}, \sum_{j=1}^{3} Q_{j}^{2} r_{0}^{j}+q^{2}, \sum_{j=1}^{3} Q_{j}^{3} r_{0}^{j}+q^{3}\right) d^{3} \mathbf{r}_{0} .
\end{aligned}
$$

This Lagrangian coincides with (2.17), so it is transformed also into the form (2.19)-(2.21). Therefore Theorem 2 is proven by the induction.

The integrability of the dynamics of the mass center $\mathbf{q}(t)$ in terms of elementary functions and the integrability of rotations of all rigid bodies $T_{\alpha}$ in terms of the Riemann theta-functions obviously follow from the separation of variables in the Lagrangian $L_{S^{k}}(3.1)$ and from the results of works $[6,7]$, see also the next section.

Remark 2. Let some $C^{k}$-central configuration $S^{k}$ with the main rigid body $T_{0 k}$ be coupled with another system of rigid bodies $S$ by one spherical joint, belonging to the rigid body $T_{0 k}$. Then the Lagrangian of the whole system $S \cup S^{k}$ has the form

$$
L_{S \cup S^{k}}=L_{S}+L_{S, T_{k}}+\sum_{\alpha=1}^{N-1} L_{2 \alpha}\left(Q_{\alpha}, \dot{Q}_{\alpha}\right) .
$$

Here $L_{S}$ is the Lagrangian of the system $S$, function $L_{S, T_{k}}$ describes interaction between system $S$ and the effective rigid body $T_{k}$. The Lagrangians $L_{2 \alpha}\left(Q_{\alpha}, \dot{Q}_{\alpha}\right)$ of all other $N-1$ rigid bodies of the $C^{k}$-central configuration $S^{k}$ are separated (in the Newtonian gravitational field with quadratic potential). Rotations of these $N-1$ rigid bodies around their spherical joints are described by the independent Lagrange system with the Lagrangians of the form (3.3).

Remark 3. The construction used for the effective rigid bodies $T_{i}$ does not coincide with that of the augmented rigid bodies, introduced by Wittenburg [26]. For example, all the augmented rigid bodies have equal masses, but effective rigid bodies $T_{i}$ have different masses.

\section{Hidden Symmetry of the Inertial Dynamics}

I. The inertial dynamics of a $C^{k}$-central configuration $S^{k}$ is described in view of Theorem 2 by the Lagrange system with the Lagrangian

$$
L_{S^{k}}=\frac{1}{2} m(\dot{\mathbf{q}}, \dot{\mathbf{q}})-\frac{1}{2} \sum_{\alpha=1}^{N} \operatorname{Tr}\left(J_{\alpha} Q_{\alpha}^{-1} \dot{Q}_{\alpha} Q_{\alpha}^{-1} \dot{Q}_{\alpha}\right),
$$

coinciding with the kinetic energy. The rotational part of the Lagrangian (4.1) has a large group of hidden symmetries $G=\prod_{\alpha=1}^{N} S O(3)_{\alpha}$, acting by the left multiplications

$$
Q_{\alpha} \rightarrow R_{\alpha} Q_{\alpha}, \quad R_{\alpha} \in S O(3)_{\alpha} .
$$

In view of the explicit form of the Lagrangian (4.1) all rigid bodies of the $C^{k}$-central configurations $S^{k}$ rotate around their spherical joints independently. For example there are stable regimes of dynamics in which certain $K$ effective rigid 
bodies inertially rotate and other $N-K$ rigid bodies do not rotate and move in space remaining parallel to themselves.

The Lagrangian (4.1) determines the left-invariant metric on the configuration space $M$, which is the direct product of Lie groups

$$
M=R^{3} \times \prod_{\alpha=1}^{N} S O(3)_{\alpha}
$$

and has symmetry group $E_{3} \times G$, where $E_{3}$ is the group of all Euclidean motions of the space $R^{3}$. The corresponding momentum map, see Abraham and Marsden [1], has the form

$$
\left(q, \dot{q}, Q_{\alpha}, \dot{Q_{\alpha}}\right) \rightarrow\left(\mathbf{p}=m \dot{\mathbf{q}}, \quad \bar{M}_{\alpha}=Q_{\alpha} M_{\alpha} Q_{\alpha}^{-1}\right) .
$$

The vector $\mathbf{p}$ and $N$ skew-symmetric matrices $\bar{M}_{\alpha}$ are first integrals of the Lagrange system. Skew-symmetric matrices of angular velocity $\omega_{\alpha}$ and angular momentum $m_{\alpha}$ in the rotating frames of reference, connected with the $\alpha^{\text {th }}$ effective rigid body, are determined by the formulae

$$
\begin{aligned}
\omega_{\alpha} & =Q_{\alpha}^{-1} \dot{Q}_{\alpha}, \\
M_{\alpha} & =J_{\alpha} \omega_{\alpha}+\omega_{\alpha} J_{\alpha}, \\
J_{\alpha i j} & =\frac{1}{2} \operatorname{Tr}\left(I_{\alpha}\right) \delta_{i j}-I_{\alpha i j} .
\end{aligned}
$$

Here $I_{\alpha i j}$ are entries of the inertia tensor of the $\alpha^{\text {th }}$ effective rigid body. Lagrangian equations with the Lagrangian $L_{S^{k}}$ (4.1) admit the reduction to the Euler equations on the Lie coalgebra $\mathscr{A}_{3 N}=\bigoplus_{\alpha=1}^{N} S O(3)_{\alpha}$ :

$$
\dot{M}_{\alpha}=\left[M_{\alpha}, \omega_{\alpha}\right], \quad \alpha=1, \ldots, N .
$$

These equations have $2 N$ independent and involutive first integrals

$$
I_{1 \alpha}=\frac{1}{2} \operatorname{Tr}\left(M_{\alpha}^{2}\right), \quad I_{2 \alpha}=\frac{1}{2} \operatorname{Tr}\left(M_{\alpha} \omega_{\alpha}\right) .
$$

The total kinetic energy of the inertial dynamics of the $C^{k}$-central configuration has the form

$$
T=\frac{1}{2} m(\dot{\mathbf{q}}, \dot{\mathbf{q}})-\frac{1}{2} \sum_{\alpha=1}^{N} \operatorname{Tr}\left(M_{\alpha} \omega_{\alpha}\right) .
$$

Inertial dynamics of all rigid bodies in the $C^{k}$-central configuration is integrable in terms of the elliptic functions.

Remark 4. The momentum map (4.4) determines $3 N+3$ adiabatic invariants of the inertial dynamics of system $S$ of $N$ coupled rigid bodies which is a small perturbation of some $C^{k}$-central configuration. Inertial dynamics of such systems, which is described by dynamics of geodesics of certain metrics on the configuration space $M(4.3)$ can be studied by the methods of the Kolmogorov-Arnold-Moser theory.

II. Dynamics of a $C^{k}$-central configuration in a Newtonian gravitational field with an arbitrary quadratic potential $\varphi(\mathbf{q})(2.4)$ is described by the Lagrange system with 
the Lagrangian (3.1). This system is equivalent to the split system of matrix equations

$$
\dot{M}_{\alpha}=\left[M_{\alpha}, \omega_{\alpha}\right]+\left[u_{\alpha}, J_{\alpha}\right], \quad \dot{u}_{\alpha}=\left[u_{\alpha}, \omega_{\alpha}\right],
$$

where $u_{\alpha}=Q_{\alpha}^{t} a Q_{\alpha}$ and the Lagrange equations for the dynamics of the mass center $\mathbf{q}(t)$ with the Lagrangian $L_{1}=\frac{1}{2} m(\dot{\mathbf{q}}, \dot{\mathbf{q}})-m \varphi(\mathbf{q})$.

Equations (4.8), as shown by Bogoyavlenskij $[6,7]$ are the Euler equations on the dual space to the Lie algebra $A_{9}$ and have the Hamiltonian

$$
H_{\alpha}=\frac{1}{2} \operatorname{Tr}\left(M_{\alpha} \omega_{\alpha}\right)+\operatorname{Tr}\left(J_{\alpha} u_{\alpha}\right)
$$

Vectors $a$ of the Lie algebra $A_{9}$ have the form $a=M+u$, where $M$ is a skewsymmetric $3 \times 3$ matrix and $u$ is a symmetric $3 \times 3$ matrix. Commutators of these matrices are determined by the formulae

$$
\begin{aligned}
{\left[M_{1}, M_{2}\right] } & =M_{1} M_{2}-M_{2} M_{1}, \\
{[M, u] } & =M u-u M, \\
{\left[u_{1}, u_{2}\right] } & =0 .
\end{aligned}
$$

Thus the Lie algebra $A_{9}$ is the semidirect sum $S O(3) \oplus R^{6}$.

Therefore the discussed Lagrange system has the reduction to the Euler equations on the Lie coalgebra $\mathscr{A}_{9 N}^{*}$, where $\mathscr{A}_{9 N}=\bigoplus_{\alpha=1}^{N} A_{9}$.

Systems (4.8) are equivalent to the Lax equations with spectral parameter $E$ :

$$
\begin{aligned}
& \dot{L}_{\alpha}=\left[L_{\alpha}, A_{\alpha}\right], \\
& L_{\alpha}=J_{\alpha}^{2} E^{2}+M_{\alpha} E+u_{\alpha}, \\
& A_{\alpha}=J_{\alpha} E+\omega_{\alpha} .
\end{aligned}
$$

Therefore $N$ Riemann surfaces $\boldsymbol{\Gamma}_{\alpha}$, determined by equations

$$
R_{\alpha}(w, E)=\operatorname{det}\left(J_{\alpha}^{2} E^{2}+M_{\alpha} E+u_{\alpha}-w \cdot 1\right)=0
$$

are associated with trajectories of the systems (4.8). All coefficients of Eq. (4.12) are involutive first integrals of (4.8), for example, $3 N$ independent first integrals

$$
I_{1 \alpha}=H_{\alpha}, \quad I_{2 \alpha}=\operatorname{Tr}\left(M_{\alpha}^{2}+2 J_{\alpha}^{2} u_{\alpha}\right), \quad I_{3 \alpha}=\operatorname{Tr}\left(M_{\alpha}^{2} u_{\alpha}+J_{\alpha}^{2} u_{\alpha}^{2}\right) .
$$

Trajectories of systems (4.8) are integrable in terms of the theta-functions of Riemann surface $\boldsymbol{\Gamma}_{\alpha}$, see works $[6,7]$.

Three-dimensional dynamics of each rigid body, belonging to the $C^{k}$-central configuration $S^{k}$, is integrable in terms of the theta-functions of several Riemann surfaces $\boldsymbol{\Gamma}_{\alpha}$, the number of which is equal to the number of rigid bodies, coupling the chosen rigid body with the main rigid body $T_{0 k}$.

Remark 5. Three-dimensional dynamics of a system $S$ of $N$ coupled rigid bodies, which is a small perturbation of a $C^{k}$-central configuration $S^{k}$, in the Newtonian gravitational field with quadratic potential $\varphi(\mathbf{q})(2.4)$, possesses $3 N$ adiabatic 
invariants $I_{1 \alpha}, I_{2 \alpha}, I_{3 \alpha}$ (4.13). Dynamics of such a system $S$ can be studied by the methods of the Kolmogorov-Arnold-Moser theory.

\section{Reductions and Integrable Cases of $C^{k}$-Central Configuration Rotation Around a Fixed Point in the Newtonian Gravitational Fields with Quadratic and Linear Potentials}

In this section we consider rotation of some $C^{k}$-central configuration $S^{k}$ with the main rigid body $T_{0 k}$ around a fixed point $P_{0}$, belonging to $T_{0 k}$. Let the origin of the Lagrange coordinates $r_{0}^{1}, r_{0}^{2}, r_{0}^{3}$ (for particles of the rigid body $T_{0 k}$ ) and the origin of the Euler coordinates $x^{1}, x^{2}, x^{3}$ coincide with the fixed point $P_{0}$, so the corresponding vector $\mathbf{q}(t)$ has coordinates $(0,0,0)$.

The Lagrangian of the $C^{k}$-central configuration $S^{k}$ in the Newtonian gravitational field with quadratic potential (2.4) has the form (3.8), where Lagrangian $L_{T_{k}}$ is calculated by the formula (3.9) with $q^{i}(t) \equiv 0$. The mass center of the effective rigid body $T_{k}$ has Lagrange coordinates

$$
R^{i}=\int_{T_{k}} \rho\left(\mathbf{r}_{0}\right) r_{0}^{i} d^{3} \mathbf{r}_{0} .
$$

The inertia tensor $I_{k i j}$ is calculated with respect to the fixed point $P_{0}$ :

$$
\begin{aligned}
J_{k i j} & =\frac{1}{2} \operatorname{Tr}\left(I_{k}\right) \delta_{i j}-I_{k i j} \\
& =\int_{T_{k}} \rho\left(\mathbf{r}_{0}\right) r_{0}^{i} r_{0}^{j} d^{3} \mathbf{r}_{0} .
\end{aligned}
$$

In these notations the Lagrangian $L_{T_{k}}$ (3.9) has the form

$$
L_{T_{k}}=-\frac{1}{2} \operatorname{Tr}\left(J_{k} Q^{-1} \dot{Q} Q^{-1} \dot{Q}\right)+\frac{1}{2} \operatorname{Tr}\left(Q^{t} a Q I_{k}\right)-\frac{1}{2}(\mathbf{b}, Q \mathbf{R}) .
$$

Corollary 1. In the considered problem all $N$ effective rigid bodies of the $C^{k}$-central configuration $S^{k}$ rotate around their spherical joints independently. Rotation of each of the $N-1$ rigid bodies, except the effective rigid body $T_{k}$, is described by the integrable Lagrange systems with Lagrangians $L_{2 \alpha}\left(Q_{\alpha}, \dot{Q}_{\alpha}\right)$ of the form (3.3). Dynamics of the configuration $S^{k}$ possesses $3(N-1)$ involutive first integrals (4.13), where $\alpha=1, \ldots, N-1$.

Corollary 2. Rotation of the effective rigid body $T_{k}$ is integrable in the following cases:

1) Fixed point $P_{0}$ coincides with the mass center of the effective rigid body $T_{k}$, that means $R^{i}=0$.

2) Fixed point $P_{0}$ is arbitrary, but potential $\rho\left(x^{1}, x^{2}, x^{3}\right)$ is pure quadratic, that means $b_{i}=0$.

In both these cases the Lagrangian (5.3) coincides with the integrable Lagrangian (3.5) and dynamics of all configuration $S^{k}$ possesses $3 N$ involutive first integrals (4.13), where $\alpha=1, \ldots, N$.

Corollary 3. Dynamics of an arbitrary $C^{k}$-central configuration $S^{k}$ around a fixed point in the Newtonian gravitational field with linear potential $\varphi\left(x^{1}, x^{2}, x^{3}\right), a_{i j} \equiv 0$, 
possesses a large group of hidden symmetries, $G_{1}=\prod_{\alpha=1}^{N-1} S O(3)_{\alpha}$, acting as in (3.10). The momentum map has the form (4.4), where $\mathbf{p}=0, \alpha=1, \ldots, N-1$. Rotation of each of the $N-1$ effective rigid bodies is pure inertial and is described by geodesics of the left-invariant metrics on the Lie group $\mathrm{SO}(3)_{\alpha}$ with the Lagrangian

$$
L=-\frac{1}{2} \operatorname{Tr}\left(J_{\alpha} Q_{\alpha}^{-1} \dot{Q}_{\alpha} Q_{\alpha}^{-1} \dot{Q}_{\alpha}\right)
$$

Rotation of the effective rigid body $T_{k}$ is described by the Lagrangian

$$
L=-\frac{1}{2} \operatorname{Tr}\left(J Q^{-1} \dot{Q} Q^{-1} \dot{Q}\right)-\frac{1}{2}(\mathbf{b}, Q \mathbf{R}) .
$$

The corresponding Lagrange equations are reduced to the Euler-Poisson equations which are integrable only in three cases, discovered by Euler $\left(R^{i}=0\right)$, Lagrange $\left(I_{1}=I_{2}, R^{1}=R^{2}=0\right)$ and Kovalevskaya $\left(I_{1}=I_{2}=2 I_{3}, R^{3}=0\right)$.

The previous considerations lead to the consequence.

Corollary 4. The problem of rotation of an arbitrary $C^{k}$-central configuration $S^{k}$ of $N$ coupled rigid bodies around a fixed point in the Newtonian gravitational field with quadratic or linear potential $\varphi\left(x^{1}, x^{2}, x^{3}\right)$ is reduced to the classical problem of rotation of only one effective rigid body $T_{k}$ around a fixed point. Dynamics of the whole $C^{k}$-central configuration $S^{k}$ is integrable if and only if rotation of the effective rigid body $T_{k}$ is integrable.

\section{Multibody Integrable Generalization of the Neumann Problem}

The classical Neumann problem is concerned with the dynamics of a material point on the surface of a sphere

$$
\left(q^{1}\right)^{2}+\left(q^{2}\right)^{2}+\left(q^{3}\right)^{2}=R_{0}^{2}
$$

in a field with homogeneous quadratic potential

$$
\varphi\left(x^{1}, x^{2}, x^{3}\right)=\frac{1}{2} \sum_{i, j=1}^{3} a_{i j} x^{i} x^{j} .
$$

The Neumann problem is completely integrable by the Hamilton-Jacobi method of separation of variables, see Neumann [20], Moser [19].

The following theorem describes generalization of the Neumann problem.

Theorem 3. Dynamics of an arbitrary $C^{k}$-central configuration $S^{k}$ in the Newtonian gravitational field with the homogeneous quadratic potential (6.2) under constraint that the mass center $\mathbf{P}$ of the whole configuration $S^{k}$ moves on the sphere (6.1) is completely integrable. Rotations of all rigid bodies around their spherical joints and dynamics of the mass center $\mathbf{P}$ are described by the separated integrable Lagrange systems.

Proof. In view of Theorem 2 dynamics of the $C^{k}$-central configuration $S^{k}$ is described by the Lagrangian $L_{S^{k}}$ (3.1), which is split into the sum of the separated Lagrangians $L_{1}(\mathbf{q}, \dot{\mathbf{q}})$ and $L_{2 \alpha}\left(Q_{\alpha}, \dot{Q}_{\alpha}\right)$. The Lagrangians $L_{2 \alpha}\left(Q_{\alpha}, \dot{Q}_{\alpha}\right)$ (3.3) describe 
integrable rotation of the $\alpha^{\text {th }}$ effective rigid body around the corresponding spherical joint. The Lagrangian $L_{1}$ (3.2) describes dynamics of the mass center $\mathbf{P}$, which is supposed to move on the sphere (6.1). The Lagrangian $L_{1}$ coincides with the Lagrangian of the integrable Neumann problem. Therefore the dynamics of the mass center $\mathbf{P}$ is integrable by the Hamilton-Jacobi method, and rotation of $\alpha^{\text {th }}$ rigid body is integrable in terms of the theta-functions of Riemann surface $\boldsymbol{\Gamma}_{\alpha}$, determined by Eq. (4.11).

\section{Separation of Rotations of an Orbiting Space Station Type $C^{k}$-Central Configuration}

I. Let us consider the problem of rotation of an artificial satellite of the Earth around its mass center. We suppose the gravitational field of the Earth is spherically-symmetric with the potential $\varphi=-G m_{0}|\mathbf{r}|^{-1}$, where $G$ is the gravity constant and $m_{0}$ is the mass of the Earth. It is known that the orbit of the satellite mass center is an ellipse with high accuracy and the Earth is in one of its focuses. We suppose that the origin of the fixed frame of references $x_{0}^{1}, x_{0}^{2}, x_{0}^{3}$ coincides with the center of the Earth, the satellite orbit lies in the plane $x^{3}=0$, and $\mathbf{q}(t)=\left(q^{1}(t), q^{2}(t), q^{3}(t)=0\right)$ is the vector of the satellite mass center $\mathbf{P}$ and $\gamma(t)=\frac{\mathbf{q}(t)}{|\mathbf{q}(t)|}$.

Let $x^{1}, x^{2}, x^{3}$ be local Cartesian coordinates with the origin in the mass center P: $x^{i}=x_{0}^{i}-q^{i}(t)$. The potential energy of the satellite in the gravitational field of the Earth, having potential $\varphi=-G m_{0}|\mathbf{r}|^{-1}$, is equal

$$
U=-\int_{T} \frac{G m_{0} \rho\left(\mathbf{r}_{0}\right)}{\left|\mathbf{r}\left(\mathbf{r}_{0}\right)\right|} d^{3} \mathbf{r}_{0},
$$

where $\mathbf{r}_{0}$ are Lagrange coordinates of particles of the satellite $T$. We assume that the scales $l$ of the satellite are much smaller than the distance to the Earth: $l \ll|\mathbf{q}|$. For the function $|\mathbf{r}|$ we have

$$
\begin{aligned}
|\mathbf{r}| & =(\mathbf{q}+\mathbf{x}, \mathbf{q}+\mathbf{x})^{\frac{1}{2}} \\
& =|\mathbf{q}|\left(1+\frac{2}{|\mathbf{q}|}(\gamma, \mathbf{x})+\frac{1}{|\mathbf{q}|^{2}}(\mathbf{x}, \mathbf{x})\right)^{\frac{1}{2}},
\end{aligned}
$$

where $(\mathbf{x}, \mathbf{y})$ is the scalar product. The Taylor expansion of $\frac{1}{|\mathbf{r}|}$ in the neighbourhood of the point $q^{1}(t), q^{2}(t), q^{3}(t)$ has the form

$$
\frac{1}{|\mathbf{r}|}=\frac{1}{|\mathbf{q}|}-\frac{1}{|\mathbf{q}|^{2}}(\gamma, \mathbf{x})+\frac{1}{|\mathbf{q}|^{3}}\left(3(\gamma, \mathbf{x})^{2}-\frac{1}{2}(\mathbf{x}, \mathbf{x})\right)+\mathcal{O} \frac{1}{|\mathbf{q}|^{4}} .
$$

Substituting this expression into (7.1) and integrating with respect to the Lagrange coordinates $\mathbf{r}_{0}$ we get

$$
U=-\frac{G m m_{0}}{|\mathbf{q}|}-\frac{G m_{0}}{|\mathbf{q}|^{3}} \int_{T} \rho\left(\mathbf{r}_{0}\right)\left(3(\gamma, \mathbf{x})^{2}-\frac{1}{2}(\mathbf{x}, \mathbf{x})\right) d^{3} \mathbf{r}_{0}+\mathcal{O} \frac{1}{|\mathbf{q}|^{4}} .
$$


Here we use that in view of the definition of the mass center the equality holds

$$
\int_{T} \rho\left(\mathbf{r}_{0}\right)(\gamma, \mathbf{x}) d^{3} \mathbf{r}_{0}=0 .
$$

Expression (7.4) for the potential energy is used often, see for example Beletskij [5], Modi and Suleman [17], Saruchev [24].

In view of the formula (7.4) the Lagrangian $L$ of the satellite in the gravitational field of the Earth in the main approximation has the form

$$
L=L_{1}+L_{2} \text {. }
$$

Here $L_{1}$ is the Lagrangian of the Kepler problem for dynamics of the satellite mass center $\mathbf{q}(t)$ around the Earth

$$
L_{1}=\frac{1}{2} m(\dot{\mathbf{q}}, \dot{\mathbf{q}})+\frac{G m m_{0}}{|\mathbf{q}|} .
$$

Lagrangian $L_{2}$ describes rotation of the satellite around its mass center in the nonstationary gravitational field with homogeneous quadratic potential

$$
\varphi\left(x^{1}, x^{2}, x^{3}\right)=-\frac{G m_{0}}{|\mathbf{q}(t)|^{3}} \sum_{i, j=1}^{3}\left(3 \gamma^{i}(t) \gamma^{j}(t)-\frac{1}{2} \delta_{i j}\right) x^{i} x^{j}
$$

II. Assume now the artificial satellite is a space station type $C^{k}$-central configuration $S^{k}$ of $N$ coupled rigid bodies. The mass center of such configuration does not move if all $N-1$ rigid bodies rotate arbitrarily around their spherical joints and the main effective rigid body $T_{k}$ rotates around its mass center. The method of investigation of the Lagrangian $L_{S^{k}}$ of the $C^{k}$-central configuration $S^{k}$ developed for the proof of Theorems 1 and 2 in Sects. 2 and 3 is applicable also when the quadratic gravitational potential (2.4) is a nonstationary one and has the form (7.8), for example. Therefore we prove by the same method the following result.

Theorem 4. The Lagrangian $L_{S^{k}}$, describing rotation of a $C^{k}$-central configuration $S^{k}$ around its mass center, moving around the Earth on the orbit $\mathbf{q}(t)$, is split into the sum

$$
L_{S^{k}}=\sum_{\alpha=1}^{N} L_{\alpha} .
$$

Lagrangians $L_{\alpha}$ have the form

$$
L_{\alpha}=-\frac{1}{2} \operatorname{Tr}\left(J_{\alpha} Q_{\alpha}^{-1} \dot{Q}_{\alpha} Q_{\alpha}^{-1} \dot{Q}_{\alpha}\right)+\frac{1}{2} \operatorname{Tr}\left(Q_{\alpha}^{t} a Q_{\alpha} I_{\alpha}\right)
$$

where matrix $a$ is nonstationary with the entries

$$
a_{i j}(t)=-\frac{3 G m_{0}}{|\mathbf{q}(t)|^{3}} \gamma^{i}(t) \gamma^{j}(t), \quad \gamma(t)=\frac{\mathbf{q}(t)}{|\mathbf{q}(t)|} .
$$

In view of the separation of rotations in Lagrangian $L_{S^{k}}$ (7.9) each effective rigid body $T_{\alpha}$ from the $C^{k}$-central configuration $S^{k}$ rotate around its spherical joint independently, as well as the main effective rigid body $T_{k}$ rotates around its mass center. Therefore we obtain the following consequence. 
Corollary 5. The problem of stabilization of rotations may be solved independently for each rigid body $T_{\alpha}$ from the orbiting space station type $C^{k}$-central configuration $S^{k}$.

III. Let $\Omega=\dot{\varphi}=\mu|\mathbf{q}(t)|^{-2}$ be the angular velocity of the mass center $\mathbf{q}(t), \mu$ is its orbital angular momentum. By definition we have

$$
\begin{aligned}
\gamma^{1}(t) & =\cos \varphi(t), \\
\gamma^{2}(t) & =\sin \varphi(t), \\
\gamma^{3}(t) & =0, \\
\frac{d \gamma}{d t} & =-\Omega \gamma \times \mathbf{n}, \\
\Omega & =\dot{\varphi},
\end{aligned}
$$

where $\mathbf{n}$ is the constant unit vector, orthogonal to the orbit $\mathbf{q}(t)$.

For the matrix $\bar{a}_{i j}=\gamma_{i} \gamma_{j}$ we have

$$
\begin{gathered}
\bar{a}=Q_{1} e Q_{1}^{t}, \\
\dot{Q}_{1}=\Omega n_{0} Q_{1},
\end{gathered}
$$

where

$$
Q_{1}=\left(\begin{array}{ccc}
\cos \varphi & -\sin \varphi & 0 \\
\sin \varphi & \cos \varphi & 0 \\
0 & 0 & 1
\end{array}\right), \quad e=\left(\begin{array}{lll}
1 & 0 & 0 \\
0 & 0 & 0 \\
0 & 0 & 0
\end{array}\right), \quad n_{0}=\left(\begin{array}{crc}
0 & -1 & 0 \\
1 & 0 & 0 \\
0 & 0 & 0
\end{array}\right)
$$

Hence the matrices

$$
\begin{aligned}
u_{\alpha} & =-Q_{\alpha}^{t} \bar{a} Q_{\alpha} \\
& =-Q_{\alpha}^{t} Q_{1} e Q_{1}^{t} Q_{\alpha}, \\
n_{\alpha} & =Q_{\alpha}^{t} n_{0} Q_{\alpha}
\end{aligned}
$$

satisfy the equations $\left(\dot{Q}_{\alpha}=Q_{\alpha} \omega_{\alpha}\right)$

$$
\dot{u}_{\alpha}=\left[u_{\alpha}, \omega_{\alpha}-\Omega n_{\alpha}\right], \quad \dot{n}_{\alpha}=\left[n_{\alpha}, \omega_{\alpha}\right] .
$$

Therefore the Lagrange equations with the Lagrangian $L_{\alpha}$ (7.10) are embedded into the matrix equations, generalizing (4.8):

$$
\begin{aligned}
\dot{M}_{\alpha} & =\left[M_{\alpha}, \omega_{\alpha}\right]-a_{0}\left[u_{\alpha}, I_{\alpha}\right], \\
\dot{u}_{\alpha} & =\left[u_{\alpha}, \omega_{\alpha}-\Omega n_{\alpha}\right], \\
\dot{n}_{\alpha} & =\left[n_{\alpha}, \omega_{\alpha}\right], \\
a_{0} & =\frac{3 G m_{0}}{|\mathbf{q}(t)|^{3}}, \\
\Omega & =\frac{\mu}{|\mathbf{q}(t)|^{2}} .
\end{aligned}
$$


The Lagrange equations on the Lie group $S O(3)$, determined by the Lagrangian $L_{\alpha}(7.10)$ are embedded also into the system of vector equations

$$
\begin{aligned}
\dot{\mathbf{M}}_{\alpha} & =\mathbf{M}_{\alpha} \times \omega_{\alpha}+a_{0} \gamma_{\alpha} \times I_{\alpha} \gamma_{\alpha}, \\
\dot{\gamma}_{\alpha} & =\gamma_{\alpha} \times\left(\omega_{\alpha}-\Omega \mathbf{n}_{\alpha}\right), \\
\dot{\mathbf{n}}_{\alpha} & =\mathbf{n}_{\alpha} \times \omega_{\alpha} .
\end{aligned}
$$

Here all vectors $\mathbf{M}_{\alpha}, \omega_{\alpha}, \gamma_{\alpha}, \mathbf{n}_{\alpha}$ are considered in the rotating frame of reference, connected with the effective rigid body $T_{\alpha}$, its angular velocity is $\omega_{\alpha}$, its angular momentum is $\mathbf{M}_{\alpha}=I_{\alpha} \omega_{\alpha}$.

Equations (7.15) and (7.16) for the circular orbits of the mass center are autonomous, in this case $a_{0}=3 \Omega^{2}$.

IV. Let us consider the Lie algebra $L_{9}$, which is the semi-direct sum of Lie algebras $S O(3)+R^{3}+R^{3}$ with basis $X_{i}, Y_{j}^{\beta},(i, j=1,2,3, \beta=1,2)$, and commutator relations

$$
\left[X_{i}, X_{j}\right]=\varepsilon_{i j k} X_{k}, \quad\left[X_{i}, Y_{j}^{\beta}\right]=\varepsilon_{i j k} Y_{k}^{\beta}, \quad\left[Y_{i}^{\alpha}, Y_{j}^{\beta}\right]=0 .
$$

Vectors of the dual space $L_{9}^{*}$ are represented in the form $\mathbf{M}+\gamma+\mathbf{n}$, according to the decomposition indicated. The Euler equations with the Hamiltonian $H(\mathbf{M}, \gamma, \mathbf{n})$ in the space $L_{9}^{*}$ have the form

$$
\begin{aligned}
\dot{\mathbf{M}} & =\mathbf{M} \times \frac{\partial H}{\partial \mathbf{M}}+\gamma \times \frac{\partial H}{\partial \gamma}+\mathbf{n} \times \frac{\partial H}{\partial \mathbf{n}}, \\
\dot{\gamma} & =\gamma \times \frac{\partial H}{\partial \mathbf{M}}, \\
\dot{\mathbf{n}} & =\mathbf{n} \times \frac{\partial H}{\partial \mathbf{M}} .
\end{aligned}
$$

It is simple to check that Eqs. (7.16) have form (7.17) with the Hamiltonian

$$
H(\mathbf{M}, \gamma, \mathbf{n})=\frac{1}{2}\left(\mathbf{M}, I^{-1} \mathbf{M}\right)+\frac{a_{0}}{2}(\gamma, I \gamma)-\Omega(\mathbf{M}, \mathbf{n}) .
$$

Therefore Eqs. (7.17) are nonautonomous Euler equations on the Lie coalgebra $L_{9}^{*}$.

The Euler equations (7.16), (7.17) in view of the general theory by Arnold [2] are Hamiltonian with the Hamiltonian (7.18) on six-dimensional simplectic submanifolds $M^{6}=S O(3) \times R^{3}$, determined in the space $L_{9}^{*}$ by three geometric constraints:

$$
(\gamma, \gamma)=1, \quad(\mathbf{n}, \mathbf{n})=1, \quad(\gamma, \mathbf{n})=0 .
$$

Thus we obtain the following consequence from Theorem 4 .

Corollary 6. The equations of rotation of an arbitrary $C^{k}$-central configuration $S^{k}$ around its mass center $\mathbf{q}(t)$ moving around the Earth are split into the direct sum of the Euler equations on $N$ Lie coalgebras $L_{9}^{*}$. These Hamiltonian equations are autonomous in the case of circular orbit $\mathbf{q}(t)$ and nonautonomous for the general elliptic orbit of the mass center. 


\section{Separation of Rotations of $C R^{n}$-Central Configuration of Coupled Gyrostats}

I. In this section we study dynamics of more general configurations of coupled rigid bodies, including gyrostats. As known, gyrostat $T$ consists of a rigid body carrier $T_{0}$ and $N$ rigid rotors $T_{\alpha}$, rotating around axes $\ell_{\alpha}$, fixed in the carrier $T_{0}$. It is supposed that the mass center $\mathbf{q}_{\alpha}(t)$ of the rotor $T_{\alpha}$ lies on the axis $\ell_{\alpha}$ and its inertia tensor $I_{\alpha}$ is invariant under rotations around axis $\ell_{\alpha}$. Thus position of the mass center $\mathbf{q}(t)$ of the gyrostat $T$ and its inertia tensor $I$ do not change when all rotors $T_{\alpha}$ rotate and the carrier $T_{0}$ is fixed.

We call the $C R^{1}$-central configuration $C^{1}$ a system of rigid bodies obtained by spherical ball-in-socket coupling of some rigid body $\tilde{T}_{10}$, carrying $n_{1}$ rotors $\tilde{T}_{1 \alpha}(\alpha=1, \ldots, n)$, with $s$ gyrostats $T_{1}, \ldots, T_{S}$ (having masses $\left.m_{1}, \ldots, m_{S}\right)$. It is supposed that centers of joints coincide with mass centers of gyrostats $T_{1}, \ldots, T_{S}$, having coordinates $\mathbf{q}_{1}(t), \ldots, \mathbf{q}_{s}(t)$. Let us denote $\mathbf{P}_{1}, \ldots, \mathbf{P}_{S}$ the corresponding coupling points of the rigid body $\widetilde{T}_{10}$ or rotors $\widetilde{T}_{1 \alpha}$. Effective rigid body $T_{10}$ and effective rotors $T_{1 \alpha}$ are obtained by putting masses $m_{1}, \ldots, m_{S}$ into points $\mathbf{P}_{1}, \ldots, \mathbf{P}_{S}$. We suppose that the effective rigid body $T_{10}$ with effective rotors $T_{1 \alpha}$ is a gyrostat, so the mass center of each effective rotor $T_{1 \alpha}$ lies on its axis of rotation $\ell_{\alpha}$ and its inertia tensor $I_{1 \alpha}$ is invariant under rotations around axis $\ell_{\alpha}$.

By induction $C R^{k}$-central configuration $C^{k}$ is obtained by ideal spherical coupling of some rigid body $\tilde{T}_{k 0}$ carrying $n_{k}$ rotors $\tilde{T}_{k \alpha}(\alpha=1, \ldots, n)$ with $s_{k} C R^{k-1}$ central configurations $C_{1}^{k-1}, \ldots, C_{S_{k}}^{k-1}$ in their mass centers $\mathbf{q}_{1}(t), \ldots, \mathbf{q}_{S_{k}}(t)$. It is supposed that effective rigid body $T_{k 0}$ with effective rotors $T_{k \alpha}$ obtained by putting masses of the corresponding $C R^{k-1}$ central configurations $m_{1}, \ldots, m_{S_{k}}$ into coupling points $\mathbf{P}_{1}, \ldots, \mathbf{P}_{S_{k}}$ is a gyrostat, as in the previous construction.

Remark 6. Dynamics of coupled gyrostats where all rotors are symmetric and all spherical joints belong to carriers, but not to rotors, was studied by Wittenburg and Lilov [27]. Equilibria of a spacecraft with rotors were studied by Krishnaprasad and Berenstein [28]. The introduced $C R^{n}$-central configurations include more complicated systems of rigid bodies, where some rotors themselves play role of carriers and are coupled through spherical joints with $C R^{k}$-central configurations, $k<n$.

II. Theorem 5. Lagrangian describing dynamics of $C R^{n}$-central configuration in the Newtonian gravitational field with an arbitrary quadratic potential (2.4) is split into sum of noninteracting Lagrangians, describing rotations of effective gyrostats.

Proof. We shall prove Theorem 5 by induction, analogously to the proof of Theorem 2 in Sect. 3. On each step of the induction we study the Lagrangian of an effective gyrostat $T_{k}$ carrying $n_{k}$ effective rotors $T_{k \alpha}(\alpha=1, \ldots, n)$. The term effective means that the gyrostat has point masses, so the mass density $\rho_{k}\left(\mathbf{r}_{k}\right)$ of the carrier $T_{k 0}$ and mass densities $\rho_{k \alpha}\left(\mathbf{r}_{k \alpha}\right)$ of rotors $T_{k \alpha}$ contain $\delta$-functions and have form (2.15).

Dynamics of particles of the carrier $T_{k 0}$ in the Euler coordinates $x^{1}, x^{2}, x^{3}$ is described by the formulae

$$
x^{i}=\sum_{j=1}^{3} Q_{k j}^{i}(t) r_{k}^{j}+q_{k}^{i}(t),
$$


where matrix $Q_{k}(t)$ with entries $Q_{k j}^{i}(t)$ is orthogonal. The mass center of the effective gyrostat $T_{k}$ is in the origin $(0,0,0)$ of the Lagrange coordinates $r_{k}$, its Euler coordinates are $q_{k}^{i}(t)$.

Dynamics of the rotor $T_{k \alpha}$ is determined by the expression

$$
x^{i}=\sum_{j=1}^{n_{k}}\left(Q_{k} S_{k \alpha} R_{k \alpha}\right)_{j}^{i} r_{\alpha}^{j}+q_{\alpha}^{i}(t)
$$

Here $R_{k \alpha}(t)$ is the matrix of rotation

$$
R_{k \alpha}(t)=\left(\begin{array}{ccc}
\cos \varphi_{k \alpha} & -\sin \varphi_{k \alpha} & 0 \\
\sin \varphi_{k \alpha} & \cos \varphi_{k \alpha} & 0 \\
0 & 0 & 1
\end{array}\right) .
$$

Constant orthogonal matrix $S_{k \alpha}$ defines the orientation of the rotor $T_{k \alpha}$ in the carrier $T_{k 0}$, so $S_{k \alpha} \mathbf{e}_{3}=\ell_{k \alpha}$, where vector $\mathbf{e}_{3}=(0,0,1)$.

The mass center of the rotor $T_{k \alpha}$ is in the origin $(0,0,0)$ of the Lagrange coordinates $r_{k \alpha}^{j}$, its Euler coordinates are $q_{\alpha}^{i}(t)$. So the equalities hold

$$
\int_{T_{k \alpha}} \rho_{k \alpha}\left(\mathbf{r}_{k \alpha}\right) r_{k \alpha}^{i} d^{3} \mathbf{r}_{k \alpha}=0
$$

where $\rho_{k \alpha}\left(\mathbf{r}_{k \alpha}\right)$ is the mass density of the rotor $T_{k \alpha}$.

Lagrangian $L_{k}$ describing rotation of an effective gyrostat $T_{k}$ in the Newtonian gravitational field with an arbitrary quadratic potential (2.4) has the form

$$
L_{k}=L_{k 0}+\sum_{\alpha=1}^{n_{k}} L_{k \alpha}
$$

Here $L_{k 0}$ is Lagrangian of the carrier $T_{k 0}$. Lagrangian $L_{k \alpha}$ describes dynamics of the effective rotor $T_{k \alpha}$ and has the form

$$
\begin{aligned}
L_{k \alpha}= & \frac{1}{2} \int_{T_{k \alpha}} \rho_{k \alpha}\left(\mathbf{r}_{k \alpha}\right) \sum_{i=1}^{3}\left(\sum_{j=1}^{3}\left(\dot{U}_{k \alpha}\right)_{j}^{i} r_{k \alpha}^{j}+\dot{q}_{k \alpha}^{i}\right)^{2} d^{3} \mathbf{r}_{k \alpha} \\
& -\int_{T_{k \alpha}} \rho_{k \alpha}\left(\mathbf{r}_{k \alpha}\right) \varphi\left(\sum_{j=1}^{3}\left(U_{k \alpha}\right)_{j}^{1} r_{k \alpha}^{j}+q_{k \alpha}^{1}, \sum_{j=1}^{3}\left(U_{k \alpha}\right)_{j}^{2} r_{k \alpha}^{j}+q_{k \alpha}^{2},\right. \\
& \left.\sum_{j=1}^{3}\left(U_{k \alpha}\right)_{j}^{3} r_{k \alpha}^{j}+q_{k \alpha}^{3}\right) d^{3} \mathbf{r}_{k \alpha},
\end{aligned}
$$

where $U_{k \alpha}=Q_{k} S_{k \alpha} R_{k \alpha}$. Expression (8.6) in view of the equalities (8.4) obtains the form

$$
\begin{aligned}
L_{k \alpha} & =L_{k 1 \alpha}+L_{k 2 \alpha}, \\
L_{k 1 \alpha} & =\frac{1}{2} m_{k \alpha}\left(\dot{\mathbf{q}}_{k \alpha}, \dot{\mathbf{q}}_{k \alpha}\right)-m_{k \alpha} \varphi\left(\mathbf{q}_{k \alpha}\right),
\end{aligned}
$$

$$
L_{k 2 \alpha}=\frac{1}{2} \operatorname{Tr}\left(\left(Q_{k} S_{k \alpha} R_{k \alpha}\right)^{\cdot} \tilde{J}_{k \alpha}\left(Q_{k} S_{k \alpha} R_{k \alpha}\right)^{\bullet}\right)-\frac{1}{2} \operatorname{Tr}\left(Q_{k} S_{k \alpha} R_{k \alpha} \tilde{J}_{k \alpha}\left(Q_{k} S_{k \alpha} R_{k \alpha}\right)^{t} a\right) \text {, }
$$


where $a$ is symmetric matrix with entries $a_{i j}(2.4), m_{k \alpha}$ is the mass of the effective rotor $T_{k \alpha}$ and $\widetilde{J}_{k \alpha}$ is the symmetric matrix with entries

$$
\left(\tilde{J}_{k \alpha}\right)_{i j}=\int_{T_{k \alpha}} \rho_{k \alpha}\left(\mathbf{r}_{k \alpha}\right) r_{k \alpha}^{i} r_{k \alpha}^{j} d^{3} \mathbf{r}_{k \alpha} .
$$

Inertia tensor $I_{k \alpha}$ is connected with $\tilde{J}_{k \alpha}(8.10)$ by the relation

$$
\left(I_{k \alpha}\right)_{i j}=\operatorname{Tr}\left(\tilde{J}_{k \alpha}\right) \delta_{i j}-\left(\tilde{J}_{k \alpha}\right)_{i j}
$$

Both tensors $I_{k \alpha}, \tilde{J}_{k \alpha}$ are supposed to be invariant under rotations $R_{k \alpha}(t)$ of rotor $T_{k \alpha}$. Hence we have

$$
R_{k \alpha} \tilde{J}_{k \alpha} R_{k \alpha}^{t}=\tilde{J}_{k \alpha}, \quad R_{k \alpha} \tilde{J}_{k \alpha}=\tilde{J}_{k \alpha} R_{k \alpha} .
$$

This relation obviously implies that matrix $\tilde{J}_{k \alpha}$ is diagonal with entries $\left(\tilde{J}_{k \alpha}\right)_{i j}=\operatorname{diag}\left(B_{k \alpha^{\prime}} B_{k \alpha^{\prime}} C_{k \alpha}\right)$. In view of relations (8.12) Lagrangian $L_{k 2 \alpha}(8.9)$ takes the form

$$
\begin{aligned}
L_{k 2 \alpha}= & -\frac{1}{2} \operatorname{Tr}\left(J_{k \alpha} Q_{k}^{-1} \dot{Q}_{k} Q_{k}^{-1} \dot{Q}_{k}\right)-\frac{1}{2} \operatorname{Tr}\left(J_{k \alpha} R_{k \alpha}^{-1} \dot{R}_{k \alpha} R_{k \alpha}^{-1} \dot{R}_{k \alpha}\right) \\
& -\operatorname{Tr}\left(S_{k \alpha} \tilde{J}_{k \alpha} R_{k \alpha}^{-1} \dot{R}_{k \alpha} S_{k \alpha}^{t} Q_{k}^{-1} \dot{Q}_{k}\right)-\frac{1}{2} \operatorname{Tr}\left(Q_{k} J_{k \alpha} Q_{k}^{t} a\right)
\end{aligned}
$$

Here we denote $J_{k \alpha}=S_{k \alpha} \tilde{J}_{k \alpha} S_{k \alpha}^{t}$.

For the rotation matrix $R_{k \alpha}(t)(8.3)$ the equality holds

$$
R_{k \alpha}^{-1} \dot{R}_{k \alpha}=\dot{\varphi}_{k \alpha} \sigma, \quad \sigma=\left(\begin{array}{ccc}
0 & -1 & 0 \\
1 & 0 & 0 \\
0 & 0 & 0
\end{array}\right) .
$$

Therefore Lagrangian (8.13) is transformed into Lagrangian

$$
\begin{aligned}
L_{k 2 \alpha}= & -\frac{1}{2} \operatorname{Tr}\left(J_{k \alpha} Q_{k}^{-1} \dot{Q}_{k} Q_{k}^{-1} \dot{Q}_{k}\right)-\frac{1}{2} \operatorname{Tr}\left(Q_{k} J_{k \alpha} Q_{k}^{t} a\right) \\
& +\frac{1}{2} A_{k \alpha} \dot{\varphi}_{k \alpha}^{2}+B_{k \alpha} \dot{\varphi}_{k \alpha} \operatorname{Tr}\left(z_{k \alpha} Q_{k}^{-1} \dot{Q}_{k}\right)
\end{aligned}
$$

where $A_{k \alpha}=-\operatorname{Tr}\left(S_{k \alpha} \tilde{J}_{k \alpha} S_{k \alpha}^{t} \sigma^{2}\right)$ is constant and $z_{k \alpha}$ is constant skew-symmetric matrix

$$
z_{k \alpha}=-S_{k \alpha} \sigma S_{k \alpha}^{t} .
$$

By definition the mass centers $\mathbf{q}_{k \alpha}(t)$ of the effective rotors $T_{k \alpha}$ are immovable relative to the carrier $T_{k 0}$. Effective rigid body $T_{k 0}$ is obtained from $\widetilde{T}_{k 0}$ by putting masses $m_{k \alpha}$ of rotors $T_{k \alpha}$ into the points $\mathbf{q}_{k \alpha}(t)$. So the mass density of the effective rigid body $T_{k 0}$ is determined by the formula

$$
\rho_{k}\left(\mathbf{r}_{k}\right)=\rho_{k 0}\left(\mathbf{r}_{k}\right)+\sum_{\alpha=0}^{n_{k}} m_{k \alpha} \delta\left(\mathbf{r}_{k}-\mathbf{P}_{k \alpha}\right),
$$

where vectors $\mathbf{P}_{k \alpha}$ define positions of mass centers $\mathbf{q}_{k \alpha}$ in the Lagrange coordinates $r_{k}^{1}, r_{k}^{2}, r_{k}^{3}$. 
In view of the formula (8.5)-(8.8) Lagrangian $\tilde{L}_{k 0}$ of the effective rigid body $T_{k 0}$ has the form

$$
\begin{gathered}
\tilde{L}_{k 0}=L_{k 0}+\sum_{\alpha=1}^{n_{k}} L_{k 1 \alpha}=L_{k 1}+\tilde{L}_{k 2} \\
L_{k 1}=\frac{1}{2} m_{k}\left(\dot{\mathbf{q}}_{k}, \dot{\mathbf{q}}_{k}\right)-m_{k} \varphi\left(\mathbf{q}_{k}\right) \\
\tilde{L}_{k 2}=-\frac{1}{2} \operatorname{Tr}\left(\tilde{J}_{k} Q_{k}^{-1} \dot{Q}_{k} Q_{k}^{-1} \dot{Q}_{k}\right)-\frac{1}{2} \operatorname{Tr}\left(Q_{k} \tilde{J}_{k} Q_{k}^{t} a\right)
\end{gathered}
$$

Here $m_{k}$ is the mass of the whole gyrostat $T_{k}$, vector $\mathbf{q}_{k}(t)$ determines position of its mass center, tensor $\tilde{J}_{k}$ is defined by the formula

$$
\left(\tilde{J}_{k}\right)_{i j}=\int_{\widetilde{T}_{k 0}} \rho_{k}\left(r_{k}\right) r_{k}^{i} r_{k}^{j} d^{3} r_{k}
$$

Substituting formulae (8.18)-(8.20), (8.15) and (8.7) into the initial sum (8.5) we obtain the following expression for the Lagrangian $L_{k}$ of the gyrostat $T_{k}$ :

$$
\begin{aligned}
L_{k}= & L_{k 1}+L_{k 2}, \\
L_{k 2}= & -\frac{1}{2} \operatorname{Tr}\left(J_{k} Q_{k}^{-1} \dot{Q}_{k} Q_{k}^{-1} \dot{Q}_{k}\right)-\frac{1}{2} \operatorname{Tr}\left(Q_{k} J_{k} Q_{k}^{t} a\right) \\
& +\sum_{\alpha=1}^{n_{k}}\left(\frac{1}{2} A_{k \alpha} \dot{\varphi}_{k \alpha}^{2}+B_{k \alpha} \dot{\varphi}_{k \alpha} \operatorname{Tr}\left(z_{k \alpha} Q_{k}^{-1} \dot{Q}_{k}\right)\right),
\end{aligned}
$$

where tensor $J_{k}$ has the form

$$
J_{k}=\tilde{J}_{k}+\sum_{\alpha=1}^{n_{k}} J_{k \alpha}
$$

Lagrangian $L_{k 2}$ (8.22) has $n_{k}$ cyclic coordinates $\varphi_{k \alpha}$. Therefore applying the Routh transformation

$$
\begin{aligned}
& L_{k 3}=L_{k 2}-\sum_{\alpha=1}^{n_{k}} p_{k \alpha} \dot{\varphi}_{k \alpha}, \\
& p_{k \alpha}=\frac{\partial L_{k 2}}{\partial \dot{\varphi}_{k \alpha}}=A_{k \alpha} \dot{\varphi}_{k \alpha}+B_{k \alpha} \operatorname{Tr}\left(z_{k \alpha} Q_{k \alpha}^{-1} \dot{Q}_{k \alpha}\right),
\end{aligned}
$$

we obtain that Lagrange equations with Lagrangian $L_{k 2}(8.22)$ are equivalent to the Routh equations

$$
\begin{gathered}
\frac{\partial L_{k 3}}{\partial Q_{k i}^{j}}=\frac{d}{d t} \frac{\partial L_{k 3}}{\partial \dot{Q}_{k i}^{j}}, \\
\dot{p}_{k \alpha}=\frac{\partial L_{k 3}}{\partial \varphi_{k \alpha}}=0, \quad \dot{\varphi}_{k \alpha}=-\frac{\partial L_{k 3}}{\partial p_{k \alpha}} .
\end{gathered}
$$

The last equation (8.27) has the form

$$
\dot{\varphi}_{k \alpha}=\frac{1}{A_{k \alpha}} p_{k \alpha}-\frac{B_{k \alpha}}{A_{k \alpha}} \operatorname{Tr}\left(z_{k \alpha} Q_{k}^{-1} \dot{Q}_{k}\right) .
$$


Substituting these expressions into (8.24) we obtain the formula

$$
\begin{aligned}
L_{k 3}= & -\frac{1}{2} \operatorname{Tr}\left(J_{k} Q_{k}^{-1} \dot{Q}_{k} Q_{k}^{-1} \dot{Q}_{k}\right)-\frac{1}{2} \operatorname{Tr}\left(Q_{k} J_{k} Q_{k}^{t} a\right) \\
& -\sum_{\alpha=1}^{n_{k}}\left(\frac{B_{k \alpha}^{2}}{2 A_{k \alpha}}\left(\operatorname{Tr}\left(z_{k \alpha} Q_{k}^{-1} \dot{Q}_{k}\right)\right)^{2}+p_{k \alpha} \frac{B_{k \alpha}}{A_{k \alpha}} \operatorname{Tr}\left(z_{k \alpha} Q_{k \alpha}^{-1} \dot{Q}_{k \alpha}\right)-\frac{p_{k \alpha}^{2}}{2 A_{k \alpha}}\right) .
\end{aligned}
$$

Here $A_{k \alpha}, B_{k \alpha}, p_{k \alpha}$ are constants and $z_{k \alpha}$ is the constant skew-symmetric matrix (8.16). Therefore we get that dynamics of the effective gyrostat $T_{k}$ is described by Lagrange equations with Lagrangian

$$
\tilde{L}_{k}=L_{k 1}+L_{k 3}
$$

and Eqs. (8.28), determining the relative rotations of the effective rotors $T_{k \alpha}$.

Formula (8.30) with Lagrangians $L_{k 1}$ and $L_{k 3}$ defined by (8.19) and (8.29) shows that Lagrangian $\tilde{L}_{k}$ is split into two noninteracting parts, describing dynamics of mass center of the effective gyrostat $T_{k}$ and its rotation around mass center. Therefore the first and consequent statements of the induction are proven. Hence Lagrangian of the whole $C R^{n}$-central configuration has the form

$$
L=L_{1}+\sum_{k=1}^{N} L_{k 3} .
$$

Here Lagrangian $L_{1}=m(\dot{\mathbf{q}}, \dot{\mathbf{q}})-\varphi(\mathbf{q})$ describes dynamics of the mass center $\mathbf{q}(t)$ of the whole configuration. Lagrangians $L_{k 3}$ describe rotations of effective gyrostats and have the form (8.29). Theorem 5 is proven.

III. Lagrange equations with Lagrangian (8.29) are equivalent to the following matrix equations:

$$
\begin{aligned}
\dot{M}_{k} & =\left[M_{k}+N_{k}, \omega_{k}\right]+\left[u_{k}, J_{k}\right], \\
\dot{u}_{k} & =\left[u_{k}, \omega_{k}\right],
\end{aligned}
$$

where the notations are used

$$
\begin{aligned}
M_{k} & =J_{k} \omega_{k}+\omega_{k} J_{k}+2 \sum_{\alpha=1}^{n_{k}} \frac{B_{k \alpha}^{2}}{A_{k \alpha}} \operatorname{Tr}\left(z_{k \alpha} \omega_{k}\right) z_{k \alpha}, \\
N_{k} & =-2 \sum_{\alpha=1}^{n_{k}} \frac{B_{k \alpha}}{A_{k \alpha}} p_{k \alpha} z_{k \alpha}, \quad \omega_{k}=Q_{k}^{-1} \dot{Q}_{k}, \quad u_{k}=Q_{k}^{t} a Q_{k} .
\end{aligned}
$$

Here only skew-symmetric matrix $N_{k}$ depends on conserved momenta $p_{k \alpha}$ and is constant.

Equations (8.32) have first integral

$$
H_{k}=\frac{1}{2} \operatorname{Tr}\left(M_{k} \omega_{k}\right)+\operatorname{Tr}\left(u_{k} J_{k}\right),
$$

which does not depend on the constant matrix $N_{k}$ and momenta $p_{k \alpha}$.

IV. The proof of Theorem 5 is valid as well in the case of time-dependent gravitational potential (2.4) which has the form (7.8) for example. Therefore 
applying Theorem 5 to the problem of rotation of space station type orbiting multibody configuration we obtain the following result.

Corollary 7. Equations of rotation of an arbitrary $C R^{n}$-central configuration around its mass center $\mathbf{q}(t)$ (moving around the Earth in an elliptic orbit) are split into system of noninteracting equations describing rotation of the effective gyrostats $T_{k}$ around their mass centers.

Equations of rotation of the effective gyrostats $T_{k}$ have the form, generalizing (8.32) and (7.15),

$$
\begin{aligned}
\dot{M}_{k} & =\left[M_{k}+N_{k}, \omega_{k}\right]-a_{0}\left[u_{k}, I_{k}\right], \\
\dot{u}_{k} & =\left[u_{k}, \omega_{k}-\Omega n_{k}\right], \quad \dot{n}_{k}=\left[n_{k}, \omega_{k}\right], \\
a_{0} & =\frac{3 G m_{0}}{|\mathbf{q}(t)|^{3}}, \quad \Omega=\frac{\mu}{|\mathbf{q}(t)|^{2}},
\end{aligned}
$$

where skew-symmetric matrices $M_{k}, \omega_{k}, N_{k}$ are determined by expressions (8.33).

For a circular orbit we have $a_{0}=3 \Omega^{2}=$ const. Equations (8.35) in this case have first integral

$$
H_{k}=\frac{1}{2} \operatorname{Tr}\left(M_{k} \omega_{k}\right)-a_{0} \operatorname{Tr}\left(u_{k} I_{k}\right)-\Omega \operatorname{Tr}\left(\left(M_{k}+N_{k}\right) n_{k}\right) .
$$

Equations of rotation of the effective gyrostat $T_{k}$ have the following vector form

$$
\begin{aligned}
\dot{\mathbf{M}}_{k} & =\left(\mathbf{M}_{k}+\mathbf{N}_{k}\right) \times \omega_{k}+a_{0} \gamma_{k} \times I_{k} \gamma_{k}, \\
\dot{\gamma}_{k} & =\gamma_{k} \times\left(\boldsymbol{\omega}_{k}-\Omega \mathbf{n}_{k}\right), \quad \dot{\mathbf{n}}_{k}=\mathbf{n}_{k} \times \boldsymbol{\omega}_{k} .
\end{aligned}
$$

These equations in case of circular orbit $\left(a_{0}=3 \Omega^{2}=\right.$ const) have first integral

$$
H_{k}=\frac{1}{2}\left(\mathbf{M}_{k}, \boldsymbol{\omega}_{k}\right)+\frac{a_{0}}{2}\left(\gamma_{k}, I_{k} \gamma_{k}\right)-\Omega\left(\mathbf{M}_{k}+\mathbf{N}_{k}, \mathbf{n}_{k}\right) \text {. }
$$

V. Applying Theorem 5 to the problem of the inertial dynamics of a multibody configuration we obtain the consequence.

Corollary 8. The inertial dynamics of an arbitrary $C R^{n}$-central configuration is integrable.

Indeed, system (8.32) in the case of inertial dynamics $\left(u_{k}=0\right)$ is reduced to a one matrix equation

$$
\dot{M}_{k}=\left[M_{k}+N_{k}, \omega_{k}\right]
$$

This equation has two first integrals

$$
H_{k}=\frac{1}{2} \operatorname{Tr}\left(M_{k} \omega_{k}\right), \quad H_{k 2}=\operatorname{Tr}\left(M_{k}+N_{k}\right)^{2} .
$$

Therefore Eq. (8.39) in the space of $3 \times 3$ skew-symmetric matrices is integrable in elliptic functions. Rotation of the effective gyrostat $T_{k}$ is determined from the linear equation $\dot{Q}_{k}=Q_{k} \omega_{k}$. 
Remark 7. Lagrangians (8.22), (8.29) in the case of absence of gravitational field are left-invariant on the Lie group $S O(3) \times S^{1} \times \cdots \times S^{1}$. So inertial dynamics of an arbitrary $C R^{n}$-central configuration possesses large group $G$ of hidden symmetries

$$
G=\prod_{k=1}^{N} S O(3)_{k} \times\left(S^{1}\right)^{M}
$$

Here commutative subgroup $\left(S^{1}\right)^{M}$ acts as independent rotations of all effective rotors.

VI. By definition two rigid bodies are coupled by a universal joint if they are free to rotate around two intersecting axes $\ell_{1}, \ell_{2}$ which are fixed with respect to each other, see Wittenburg [26]. We consider system $U_{k}$ obtained by a universal joint of arbitrary many rigid bodies $T_{k \alpha}(\alpha=1, \ldots, n)$ having inertia tensors $I_{k \alpha}$ symmetric under rotations around axes $\ell_{k \alpha}$ and with mass centers lying on the axes $\ell_{k \alpha}$. Such systems $U_{k}$ of universally joint rigid bodies $T_{k \alpha}$ is obviously a particular case of a gyrostat $T_{k}$, for which carrier $\tilde{T}_{k 0}$ consists of all connected axes $\ell_{k \alpha}$ (not intersecting in general) and is massless.

Therefore dynamics of system $U_{k}$ in the Newtonian gravitational field with an arbitrary quadratic potential $(2.4)$ is described by Lagrange system with the Lagrangian $L_{k}(8.21)$, (8.22). Here the mass density of the effective rigid body $T_{k 0}$ is determined by the formula (8.17) with $\rho_{k 0}\left(r_{k}\right)=0$.

Lagrange equations for inertial dynamics of system $U_{k}$ are reduced to one matrix equation (8.39), which possesses two first integrals (8.40) and hence is integrable in elliptic functions.

We call the $C U^{n}$-central configuration of a particular case of $C R^{n}$-central configuration, where some of carriers $\widetilde{T}_{k 0}$ are massless, and so corresponding rotors $T_{k \alpha}$ are coupled by universal joints. Applying Theorem 5 we get the consequence.

Corollary 9. Equations of inertial dynamics of an arbitrary $C U^{n}$-central configuration are integrable.

Acknowledgements. I am grateful to Jerrold Marsden and William Shadwick for the invitation to work in the Fields Institute for Research in Mathematical Sciences. Thanks to Leo Jonker, Jon Thompson and Charles Himmelberg for useful discussions.

\section{References}

1. Abraham, R., Marsden, J.E.: Foundations of Mechanics. London, Amsterdam, Don Mills, Ontario, Sydney, Tokyo: Benjamin/Cummings 1978

2. Arnold, V.I.: Mathematical Methods in Classical Mechanics. Berlin, Heidelberg, New York: Springer 1983

3. Baillieul, J.: An Enumerative Theory of Equilibrium Rotations For Planar Kinematic Chains. Contemp. Math. 97, 1-10 (1989)

4. Bobenko, A.I., Reyman, A.G., Semenov-Tian-Shansky, M.A.: The Kowalewski Top 99 Years Later: A Lax Pair, Generalizations and Explicit Solutions. Commun. Math. Phys. 122, 321-354 (1989)

5. Beletskij, V.V.: Dynamics of an Artificial Satellite Around Mass Center. Moscow: Nauka 1965

6. Bogoyavlenskij, O.I.: New Integrable Problem of Classical Mechanics. Commun. Math. Phys. 94, 255-269 (1984)

7. Bogoyavlenskij, O.I.: Integrable Euler Equations Arising in Problems of Mathematical Physics. Izvestija of Academy of Sciences of USSR. 48, N5, 883-938 (1984) 
8. Bogoyavlenskij, O.I.: Some integrable cases of the Euler equations. Doklady of Academy of Sciences of USSR. 287, N5, 1105-1109 (1986)

9. Bogoyavlenskij, O.I.: Breaking Solitons. Nonlinear Integrable Equations. Moscow: Nauka 1991

10. Bogoyavlenskij, O.I.: Euler equations on Finite Dimensional Lie Coalgebras, Arising in Problems of Mathematical Physics. Russ. Math. Surv. 47, N1 (1992)

11. Krishnaprasad, P.S., Marsden, J.E.: Hamiltonian Structures and Stability for Rigid Bodies with Flexible Attachments. Archive for Rational Mechanics and Analysis. 98, N1, 71-93 (1981)

12. Krishnaprasad, P.S.: Eulerian Many-Body Problems. Contemp. Math. 97, 189-208 (1989)

13. Leimanis, E.: The General Problem of the Motion of Coupled Rigid Bodies About Fixed Point. Springer Tracts in Natural Philosophy, 7. Berlin, Heidelberg, New York: Springer 1965

14. Levis, D.R., Marsden, J.E., Ratiu, T.S., Simo, J.C.: Normalizing Connections and the EnergyMomentum Method. In: Proceedings of the CRM Workshop on Hamiltonian Systems, Transformation Groups and Spectral Transform Methods. Montreal: CRM, 1990

15. Marsden, J.E., Simo, J.C., Lewis, D., Posbergh, T.A.: Block Diagonalization and the Energy-Momentum Method. Contemp. Math. 97, 297-314 (1989)

16. Marsden, J.E., Montgomery, R., Ratiu, T.: Reduction, Symmetry and Phases in Mechanics. Memoirs of the American Mathematical Society, 88, N436. Providence, Rhode Island: AMS 1990

17. Modi, V.J., Suleman, A.: System Modes and Dynamics of the Proposed Space Station Type Configurations. Nonlinear Dynamics. 1, 379-400 (1990)

18. Modi, V.J., Ng, A., Suleman, A., Morita, Y.: Dynamics of Orbiting Multibody Systems: A Formulation with Application. AIAA-91-0998 (1991)

19. Moser, J.: Various Aspects of Integrable Hamiltonian Systems. Dynamical Systems (C.I.M.E. Summer School, Bressanone, 1978), Progress in Mathematics, Birkhäuser, 8, 233-289 (1980)

20. Neumann, C.: De Problemate Quodam Mechanico, Quod ad Primam Integralium Ultraellipticorum Classem Revocatur. J. Reine Angew. Math. 56, 46-63 (1958/59)

21. Patrick, G.W.: The Dynamics of Two Coupled Rigid Bodies in Three Space. Contemp. Math. 97, 315-336 (1989)

22. Patrick, G.W.: Nonlinear Stability of Coupled Rigid Bodies. Ph.D. Dissertation, Department of Mathematics, Berkeley: University of California, 1990

23. Routh, E.J.: Dynamics of a System of Rigid Bodies. New York: Dover Publications 1960

24. Saruchev, V.A.: Investigation of the Gravitational Stabilization System Dynamics. The Artificial Earth Satellites, 16, 10-33. Moscow: Academy of Sciences of USSR 1963

25. Sreenath, N., Oh, Y.G., Krishnaprasad, P.S., Marsden, J.E.: The dynamics of Coupled Planar Rigid Bodies. Part I: Reduction, Equilibria and Stability. Dynamics and Stability of Systems. 3, 25-49 (1988)

26. Wittenburg, J.: Dynamics of Systems of Rigid Bodies. Stuttgart: B.G. Teubner 1977

27. Wittenburg, J., Lilov, L.: Relative Equilibrium Positions and Their Stability for a Multi-Body Satellite in a Circular Orbit. Ingenieur - Archiv, 44, 269-279 (1975)

28. Krishnaprasad, P.S., Berenstein, C.A.: On the Equilibria of Rigid Spacecraft with Rotors. Systems \& Control Letters, 4, 157-163 (1984) 
